\title{
A Study on Facilitating Factors of User Convenience of Urban Parks -Based on User Evaluation of Barrier-Free Urban Parks in Japan-
}

\author{
Jiewoong Lee ${ }^{1 *}$, Takeshi Kinoshita', Eunsuk $\mathrm{Oh}^{1}$ and Bohyun Kim ${ }^{1}$ \\ ${ }^{1}$ Department of Horticulture, Graduate School of Chiba University, Chiba 271-8510, Japan \\ 도시공원의 이용 편의성 촉진 요인에 관한 연구 \\ -일본의 무장애(베리어 프리)화 된 도시공원의 이용자 평가를 바탕으로- \\ 이지웅 ${ }^{1 *} \cdot$ 키노시타타케시 $^{1} \cdot$ 오은석 $^{1} \cdot$ 김보현 $^{1}$ \\ ${ }^{1}$ 치바대학교 대학원 원예학과
}

\begin{abstract}
This study researched the cases of urban parks in Japan that has established and enforced legislation related to barrier-free(BF) prior to Korea. This was in order to verify the effect of BF improvement of park facilities to promote user convenience in urban parks and to show directions. To understand the effectiveness of BF improvement done in Japan, we conducted a consciousness survey and evaluation for the users of the parks designed/constructed under the standards of the guidelines for urban parks in Japan. BF improvement of park facilities based on the set standards to enhance park user convenience has an effect on improvement of park user convenience. However, improvements suitable for expected users' behavior including attributes of neighboring residents and characteristics of parks might be more efficient to increase user convenience. This should focus on items showing different evaluations for each park such as the slope of the entrance or width of path, rather than applying the same standards to every park. It would be necessary to place facilities based on users' behavior and characteristics, and by including playground equipment into the contents of the guidelines. It would be necessary to add not only standards of design and construction, but also a manual of maintenance/management. Such standards that can efficiently deliver information necessary for use of internal facilities of parks should also be prepared.
\end{abstract}

Key words: Barrier free new act, Urban park, User evaluation

\section{I . Introduction}

Urban dwellers concern for higher quality of life has increased with the increase in income levels. They have come to demand leisure space which is diverse, and of high quality level in their daily living environment. In this context, urban parks are the most easily approachable spaces that meet such needs. Among them, neighborhood parks have a close relation with urban dwellers daily life(Lee and Kim, 2001). However, the frequency of use of urban parks that have close ties with urban dwellers as facilities preferred by the disabled, the elderly and able bodied is considerably lower compared to the

Received on October 16, 2013. Revised on November 12, 2013.

Accepted on November 23, 2013.

*Corresponding author: jiewoong@hanmail.net demand for urban parks. This is due to problems such as location and connectivity with the means of transport, as well as social and physical barriers(Kang et al., 2007). In this case, elements such as blocking walls are referred to as Barriers. While creating an environment that makes it easier for senior citizens or the disabled to participate in society or use facilities by themselves, is referred to as Barrier Free(BF, Kawauchi, 2001). To fulfill BF, 'Convenience Improvement for the disabled, the elderly and pregnant women Act(Convenience Improvement Act)', and 'Mobility Convenience Improvement for the weak Transportation Act(Mobility Improvement Act)' were established. Though the Convenience Improvement Act includes regulations related to urban parks, it describes only broad standards of internal convenience facilities and general contents, without showing detailed standards(Kang et al., 
2007). Thus, it is necessary to reorganize national institutions to promote the use of urban parks by the vulnerable members of society such as the disabled and the elderly.

Japan is ranked first in life expectancy in the world(Shigeno, 2008), and is currently faced with an aging society problem. It has a close connection with Korea in the aspects of history, culture, and geography. It has carried out improvement on legislation and movements related to $\mathrm{BF}$, starting with the movement of making welfare villages since 1970s(Suzuki, 2006). In order to implement BF in the overall society, consolidation, and expansion of legislation culminated in the establishment of Barrier-Free New Act(hereafter referred to as New Act) in 2006. The target of the New Act was expanded to cover the whole society, including urban parks. This means that the improvement obligation of urban parks into BF that used to be excluded from the existing legislation came to have legal force(Guideline for promoting barrier-free transport and facilities for elderly and disabled in the city parks, 2008). After the establishment of the law, the Ministry of Land, Infrastructure, and Transport in charge of managing urban parks established and enforced 'Guideline for promoting barrier-free transport and facilities for elderly and disabled in the city parks(hereafter referred to as Guidelines)'. This was through an order by the Minister in charge in 2008, to increase park managers' understanding of barrier-free and standards of BF for urban parks. In order to have national-level BF movement for urban parks, it aided local governments that had poor finances and conducted 'Immediate Support Project for BF Urban Parks' for five years from 2006(Wakisaka, 2008).

Though both Korea and Japan are conducting BF improvement of urban parks, research on BF in urban parks is insufficient in Korea. Japan also lacks in research on the effects and problems of these improvements through users' evaluation on parks with improved facilities based on BF policies(Lee and Yoem, 2012).

Therefore, this study focused on the cases of urban parks in Japan that established and enforced legislation related to BF prior to Korea. This was in order to verify the effect of $\mathrm{BF}$ improvement of park facilities to promote user convenience in urban parks and to show future directions. To understand the effectiveness of BF improvement performed in Japan, we conducted a consciousness survey and evaluation of the actual users of the parks designed/constructed under the standards of the guidelines for urban parks in Japan. Results of this study are expected to be helpful as valid basic data to set up and be applied by institutions, for BF improvement of urban parks for user convenience in Korea and Japan.

\section{Materials and Methods}

\section{Research Subject and Outline}

This study set standards to select the target parks as follows:

1. The neighborhood park having close ties with urban residents' daily life.

2. The neighborhood park equipped with basic amenities such as entrance, path, multi-purpose restroom(restroom), and bulletin board(Nishimura and Asazawa, 2005), well maintained enough to meet the standards of guidelines for the use of the park. This include those with 12 facilities designated in the guidelines such as entrance, path and plaza, path, plaza with a roof, rest area, outdoor theater, concert garden, parking garage, restroom, drinking water fountain, washroom, management office, bulletin board, and signs.

3. The neighborhood park with adequate playground equipments(though not included in specifically-designated facilities in the guidelines), on the basis of play guidelines for safety of playground equipment.

4. Resident-participation-type park from its planning stage (park constructed through local residents' participation from the planning stage can draw more residents' interest and attachment)(Kim, 2006).

This study made the above conditions as its standard for selecting target parks, targeting the park administrators of all ward offices in 23 boroughs in Tokyo, which are aggressively enforcing BF. Among others, they are establishing and enforcing ordinances for making welfare villages, or guidelines of Universal Design among others. The study selected the target cases by making inquiries about parks that meet the standards of selection, and through the process of on-site confirmation.

Finally, five types of park facilities were investigated, where entrance, path, restroom, play equipments, and bulletin board 
became the standards. Four study cases were selected including Shindensakura Park(Shindensakura, 2010, 2.5ha), Nisiarai Sakae Park(Nisiarai, 2007, 1ha, Adachi Ward), Nishigahara Minano Park(Nishigahara, 2010, 2.2ha, Kita Ward) and Nakameguro Park(Nakameguro, 2002, 2.2ha, Meguro Ward) as target places.(The number in the parenthesis indicates the opening year and park size).

\section{Research Method}

This study compared the present status of facility maintenance to the standards of guidelines through the measurement of the five types of facilities. In an effort to grasp actual users' evaluation, we conducted an on-site attitude survey through a questionnaire. The questionnaire format was drawn based on the standards of the facilities stated in the guidelines and theoretical background through documentary survey.

As the subjects specified in the guidelines include all users, including senior citizens and the disabled, we conducted a survey of the parks users regardless of their physical conditions. Actual measurement of facilities was carried out on May 29, 2012, and subsequently analyzed 221 filled questionnaires (Table 1). The questionnaire survey of the park users was carried out from 9:00am to 5:00pm on May 31 and June 4 and 5 respectively. Since every subject park had multiple entrances, and paths connected to each other, the subjects for evaluation needed to be unified. We selected the study subjects based on the following standards. The entrance should face a major road side with a bulletin board installed. The road should be around the selected entrance. In case of evaluating items for each facility(items), elements judged to be necessary through guideline standards, pre-survey or literature were selected, based on five steps(1-5) evaluation method. The demands for facilities were evaluated using multiple selection response method. After the encoding process, the questionnaires were

Table 1. Number of respondents each park.

\begin{tabular}{cc}
\hline Park & Numbers \\
\hline Nishigahara & 41 \\
Nishiaraisakae & 79 \\
Sindensakura & 49 \\
Nakameguro & 52 \\
Total & 221 \\
\hline
\end{tabular}

analyzed using SPSS(Statistical Package for Social Science) Win 18.0 program. The analysis of each park was conducted through the means of items for evaluation of facilities, convenience and satisfaction, and through ANOVA we tested if the difference in population means of each park would be statistically significant. The frequency analysis was conducted on the demands for facilities, to understand users' opinions.

\section{Composition of Survey}

The questions of the survey were composed of categories including attributes of respondents, evaluation of park facilities, and demands for park facilities. In order to understand the attributes of the respondents, we included questions on; gender, age and whether there was any physical inconvenience and its type. In order to understand the behavior of park use, travel time, distance to parks and means of transport, we asked the purpose of use, standards of selecting parks and questions about use companions. We asked questions to evaluate user convenience of park facilities, satisfaction, and detailed standards, to understand the effect of BF facilities. To understand improving factors of facility convenience and inconvenience when using facilities, we included questions about demands for facilities.

\section{Results and Discussion}

\section{Users Attributes}

All park showed similar types of age group and sex, while the ratio of women in their 30s was highest(Fig. 1).

Based on the results of the 'purpose of using parks'(Table 2),

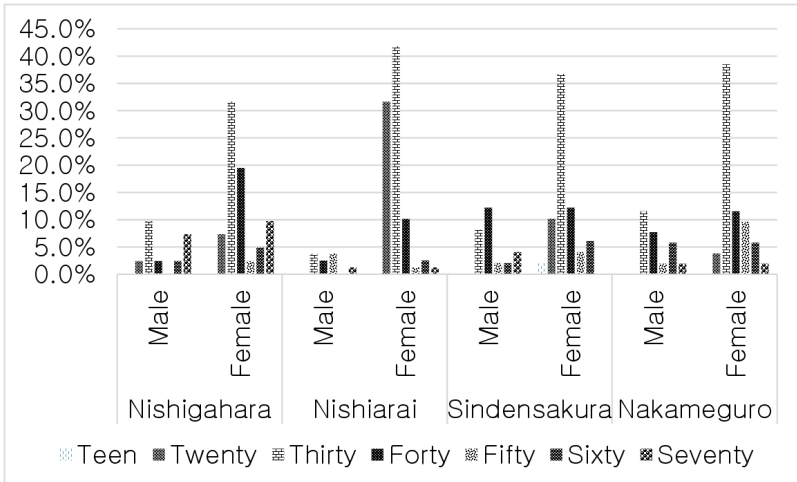

Fig. 1. User's gender and age. 
Table 2. User's Purpose of using parks(\%).

\begin{tabular}{|c|c|c|c|c|}
\hline & A & $\mathrm{B}$ & $\mathrm{C}$ & $\mathrm{D}$ \\
\hline Enjoy greenery & 4.9 & 5.1 & 12.2 & 19.2 \\
\hline walk & 24.4 & 21.5 & 34.7 & 36.5 \\
\hline To play with children & 70.7 & 92.4 & 71.4 & 48.1 \\
\hline Exercise & 9.8 & 5.1 & 14.3 & 11.5 \\
\hline Rest & 14.6 & 6.3 & 24.5 & 13.5 \\
\hline Walk a pet & 2.4 & 1.3 & 4.1 & 17.3 \\
\hline $\begin{array}{l}\text { Communicate or do hobby } \\
\text { activities with a friend }\end{array}$ & 12.2 & 5.1 & 12.2 & 9.6 \\
\hline Join event & 0.0 & 0.0 & 0.0 & 1.9 \\
\hline Rendezvous & 0.0 & 1.3 & 2.0 & 0.0 \\
\hline Others & 4.9 & 0.0 & 2.0 & 9.6 \\
\hline
\end{tabular}

A:Nishigahara, B:Nishiarai, C:Shindensakura, D:Nakameguro

Table 3. User's Companions(\%).

\begin{tabular}{lcccc}
\hline & $\mathrm{A}$ & $\mathrm{B}$ & $\mathrm{C}$ & $\mathrm{D}$ \\
\hline Alone & 14.6 & 5.1 & 18.4 & 9.6 \\
Family & 26.8 & 22.8 & 26.5 & 28.8 \\
Husband or wife & 4.9 & 0.0 & 0.0 & 3.8 \\
Kid & 46.3 & 68.4 & 44.9 & 28.8 \\
Pet & 2.4 & 2.5 & 4.1 & 15.4 \\
Friend & 4.9 & .0 & 6.1 & 9.6 \\
Others & 0.0 & 1.3 & 0.0 & 3.8
\end{tabular}

A:Nishigahara, B:Nishiarai, C:Shindensakura, D:Nakameguro

'attributes of companions(Table 3)' and high ratio of female respondents, the subject parks are mainly used by married couples and especially mothers who like to play with their children.

In all the park, majority indicated 'within ten minutes' of travel time, and the ratio of walking and bike for means of transport was more than $80 \%$ (Fig. 2). This means that most of the users were residents living close to the parks.

In regard to the reasons for selecting the park, most responses were 'close to home' $(70.7 \%, 70.9 \%, 85.7 \%$, and $53.8 \%$ ) except for Nakameguro(Table 4). This means that the element of distance has a huge influence on selecting parks, where most of the users were nearby residents. On questions about the standards of selecting parks including 'path comfortable to walk on' and 'easily recognizable circulation to the desired facilities' related to BF, only 'path comfortable to walk on' and 'possible to use play facilities safely' had $20 \%$

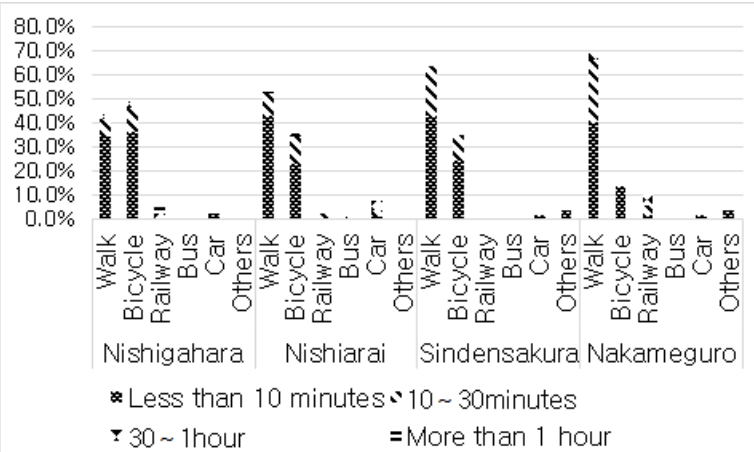

Fig. 2. Travel time and means of transportation.

Table 4. Reasons for selecting the park(\%).

\begin{tabular}{|c|c|c|c|c|}
\hline & A & $\mathrm{B}$ & $\mathrm{C}$ & $\mathrm{D}$ \\
\hline Close to home & 70.7 & 70.9 & 85.7 & 53.8 \\
\hline Abundant greens and trees & 43.9 & 13.9 & 12.2 & 69.2 \\
\hline Relatively new park & 34.1 & 36.7 & 10.2 & 9.6 \\
\hline $\begin{array}{l}\text { A pet companion is } \\
\text { possible }\end{array}$ & 4.9 & 1.3 & 4.1 & 13.5 \\
\hline Can meet a friend & 12.2 & 19.0 & 14.3 & 13.5 \\
\hline $\begin{array}{l}\text { Facilities are always } \\
\text { managed neatly }\end{array}$ & 26.8 & 31.6 & 20.4 & 30.8 \\
\hline Easy to walk a road & 24.4 & 11.4 & 26.5 & 25.0 \\
\hline Can use restroom without fear & 12.2 & 5.1 & 8.2 & 19.2 \\
\hline $\begin{array}{l}\text { Can use playground } \\
\text { equipment without fear }\end{array}$ & 22.0 & 38.0 & 26.5 & 11.5 \\
\hline Easy to use a parking area & 0.0 & 0.0 & 0.0 & 0.0 \\
\hline $\begin{array}{l}\text { Easy to use a bicycle } \\
\text { parking area }\end{array}$ & 7.3 & 3.8 & 6.1 & 1.9 \\
\hline A gentle slope & 7.3 & 3.8 & 2.0 & 7.7 \\
\hline $\begin{array}{l}\text { Line is easier to } \\
\text { understand }\end{array}$ & 4.9 & 5.1 & 4.1 & 1.9 \\
\hline Spaciousness area & 43.9 & 25.3 & 24.5 & 32.7 \\
\hline Can use a lawn plaza & 36.6 & 16.5 & 38.8 & 48.1 \\
\hline $\begin{array}{l}\text { Many kinds of park } \\
\text { facilities }\end{array}$ & 4.9 & 7.6 & 12.2 & 1.9 \\
\hline Others & 2.4 & 8.9 & 2.0 & 11.5 \\
\hline
\end{tabular}

A:Nishigahara, B:Nishiarai, C:Shindensakura, D:Nakameguro

response ratio. Other items were too low, which means that the influence of $\mathrm{BF}$ improvement on selecting parks is low. Though each subject park showed similar attributes of users, Nakameguro showed different tendency in the aspect of the 'purpose of using the park', 'companions', and 'the reason for selecting the park'. 
2. Evaluation of Park Facilities, Convenience, Satisfaction, and Differences in Evaluation for Each Park

The evaluation of facilities, convenience, satisfaction, and differences in evaluation for each park areas shown in Table 5-9.

There were differences in users' evaluation for each park facility. In every park, entrance and path received high evaluation. Regarding play facilities, Nakameguro received about 2.96 points. Considering the low evaluation of play facilities, the reason for selecting the park, and the results from observation during survey, people wanted to spend time with their children on greens instead of play facilities. This could be the cause for such behavioral difference from other parks.

There were significant differences in items that have concrete standards in guidelines such as the slope of entrance, width of the path, and width of restroom entrance among

Table 5. Evaluation of park facilities and differences in evaluation for each park(Entrance).

\begin{tabular}{|c|c|c|c|c|c|c|}
\hline Factors & & Mean & S.D & $\mathrm{F}$ & Sig. & Scheffe \\
\hline \multirow{4}{*}{ Usability } & $A$ & 3.88 & 0.980 & \multirow{4}{*}{1.429} & \multirow{4}{*}{0.235} & \multirow{4}{*}{-} \\
\hline & B & 3.82 & 0.874 & & & \\
\hline & $\mathrm{C}$ & 3.92 & 0.932 & & & \\
\hline & $\mathrm{D}$ & 3.58 & 0.893 & & & \\
\hline \multirow{4}{*}{ Width } & A & 3.88 & 0.980 & \multirow{4}{*}{3.778} & \multirow{4}{*}{0.011} & \multirow{4}{*}{-} \\
\hline & B & 3.82 & 0.874 & & & \\
\hline & $\mathrm{C}$ & 3.92 & 0.932 & & & \\
\hline & $\mathrm{D}$ & 3.58 & 0.893 & & & \\
\hline \multirow{4}{*}{ Slant } & A & 3.88 & 0.980 & \multirow{4}{*}{4.264} & \multirow{4}{*}{$0.006^{* *}$} & \multirow{4}{*}{$\mathrm{B}>\mathrm{D}$} \\
\hline & B & 3.82 & 0.874 & & & \\
\hline & $\mathrm{C}$ & 3.92 & 0.932 & & & \\
\hline & D & 3.58 & 0.893 & & & \\
\hline \multirow{4}{*}{ Surface } & A & 3.88 & 0.980 & \multirow{4}{*}{4.592} & \multirow{4}{*}{$0.004^{* *}$} & \multirow{4}{*}{-} \\
\hline & B & 3.82 & 0.874 & & & \\
\hline & $\mathrm{C}$ & 3.92 & 0.932 & & & \\
\hline & D & 3.58 & 0.893 & & & \\
\hline \multirow{4}{*}{$\begin{array}{c}\text { A } \\
\text { countermeasure } \\
\text { against vehicles } \\
\text { invasion }\end{array}$} & A & 3.88 & 0.980 & \multirow{4}{*}{1.821} & \multirow{4}{*}{0.144} & \multirow{4}{*}{-} \\
\hline & B & 3.82 & 0.874 & & & \\
\hline & $\mathrm{C}$ & 3.92 & 0.932 & & & \\
\hline & D & 3.58 & 0.893 & & & \\
\hline
\end{tabular}

The mean difference is significant at the 0.01 level.

S.D $=$ Standard Deviation. Sig $=$ Significant.

A:Nishigahara, B:Nishiarai, C:Shindensakura, D:Nakameguro others. Items excluded from the standards in guidelines such as the size of

bulletin board, items showing only broad measures like the height of bulletin board or explanations among others. This includes also playground equipments created based on the play guidelines. The user evaluation was shown differently depending on the attributes ofusers or condition of improvement(Table $5-9)$.

Table 6. Evaluation of park facilities and differences in evaluation for each park(Path).

\begin{tabular}{|c|c|c|c|c|c|c|}
\hline Factors & & Mean & S.D & $\mathrm{F}$ & Sig. & Scheffe \\
\hline \multirow{4}{*}{ Usability } & A & 4.00 & 0.949 & \multirow{4}{*}{2.953} & \multirow{4}{*}{0.034} & \multirow{4}{*}{-} \\
\hline & B & 4.10 & 0.810 & & & \\
\hline & $\mathrm{C}$ & 4.37 & 0.636 & & & \\
\hline & $\mathrm{D}$ & 3.94 & 0.639 & & & \\
\hline \multirow{4}{*}{ Width } & A & 3.90 & 0.800 & \multirow{4}{*}{6.283} & \multirow{4}{*}{$0.000^{* *}$} & \multirow{4}{*}{$\begin{array}{l}A>D \\
C>D\end{array}$} \\
\hline & B & 3.53 & 0.998 & & & \\
\hline & $\mathrm{C}$ & 3.90 & 0.848 & & & \\
\hline & $\mathrm{D}$ & 3.27 & 0.689 & & & \\
\hline \multirow{4}{*}{ Slant } & A & 3.66 & 0.855 & \multirow{4}{*}{3.499} & \multirow{4}{*}{0.016} & \multirow{4}{*}{-} \\
\hline & B & 3.80 & 0.952 & & & \\
\hline & $\mathrm{C}$ & 3.71 & 0.842 & & & \\
\hline & $\mathrm{D}$ & 3.31 & 0.781 & & & \\
\hline \multirow{4}{*}{$\begin{array}{c}\text { Road } \\
\text { surfacing } \\
\text { condition }\end{array}$} & A & 3.78 & 0.936 & \multirow{4}{*}{2.904} & \multirow{4}{*}{0.036} & \multirow{4}{*}{-} \\
\hline & $\mathrm{B}$ & 3.90 & 0.942 & & & \\
\hline & $\mathrm{C}$ & 3.78 & 0.848 & & & \\
\hline & D & 3.44 & 0.752 & & & \\
\hline \multirow{4}{*}{$\begin{array}{c}\text { Slipperiness } \\
\text { of the road } \\
\text { surface }\end{array}$} & A & 3.73 & 0.895 & \multirow{4}{*}{1.698} & \multirow{4}{*}{0.168} & \multirow{4}{*}{-} \\
\hline & B & 3.71 & 1.002 & & & \\
\hline & $\mathrm{C}$ & 3.84 & 0.874 & & & \\
\hline & $\mathrm{D}$ & 3.44 & 0.850 & & & \\
\hline \multirow{4}{*}{$\begin{array}{c}\text { Road surface } \\
\text { materials }\end{array}$} & A & 3.32 & 0.722 & \multirow{4}{*}{0.594} & \multirow{4}{*}{0.619} & \multirow{4}{*}{-} \\
\hline & B & 3.19 & 0.786 & & & \\
\hline & C & 3.35 & 0.830 & & & \\
\hline & D & 3.33 & 0.678 & & & \\
\hline \multirow{4}{*}{$\begin{array}{c}\text { Road surface } \\
\text { brightness }\end{array}$} & A & 3.76 & 0.969 & \multirow{4}{*}{5.347} & \multirow{4}{*}{$0.001^{* *}$} & \multirow{4}{*}{-} \\
\hline & B & 3.38 & 0.867 & & & \\
\hline & $\mathrm{C}$ & 3.71 & 0.791 & & & \\
\hline & $\mathrm{D}$ & 3.17 & 0.760 & & & \\
\hline
\end{tabular}

The mean difference is significant at the 0.01 level.

S.D $=$ Standard Deviation. Sig = Significant. A:Nishigahara, B:Nishiarai, C:Shindensakura, D:Nakameguro 
Table 7. Evaluation of park facilities and differences in evaluation for each park(Restroom).

\begin{tabular}{|c|c|c|c|c|c|c|}
\hline Factors & & Mean & S.D & $\mathrm{F}$ & Sig. & Scheffe \\
\hline \multirow{4}{*}{ Usability } & A & 3.63 & .888 & \multirow{4}{*}{1.318} & \multirow{4}{*}{0.270} & \multirow{4}{*}{-} \\
\hline & B & 3.28 & .960 & & & \\
\hline & $\mathrm{C}$ & 3.45 & .914 & & & \\
\hline & $\mathrm{D}$ & 3.42 & .977 & & & \\
\hline \multirow{4}{*}{$\begin{array}{l}\text { Entrance } \\
\text { width }\end{array}$} & A & 3.46 & .745 & \multirow{4}{*}{4.861} & \multirow{4}{*}{$0.003^{* *}$} & \multirow{4}{*}{$\mathrm{C}>\mathrm{D}$} \\
\hline & B & 3.28 & .715 & & & \\
\hline & $\mathrm{C}$ & 3.63 & .834 & & & \\
\hline & $\mathrm{D}$ & 3.10 & .721 & & & \\
\hline \multirow{4}{*}{$\begin{array}{l}\text { Position of a } \\
\text { doorknob }\end{array}$} & A & 3.56 & .776 & \multirow{4}{*}{0.964} & \multirow{4}{*}{0.411} & \multirow{4}{*}{-} \\
\hline & B & 3.37 & .701 & & & \\
\hline & $\mathrm{C}$ & 3.49 & .739 & & & \\
\hline & $\mathrm{D}$ & 3.35 & .738 & & & \\
\hline \multirow{4}{*}{$\begin{array}{l}\text { Method of a } \\
\text { door operator }\end{array}$} & A & 3.51 & .870 & \multirow{4}{*}{0.860} & \multirow{4}{*}{0.462} & \multirow{4}{*}{-} \\
\hline & B & 3.30 & .837 & & & \\
\hline & $\mathrm{C}$ & 3.49 & .767 & & & \\
\hline & $\mathrm{D}$ & 3.37 & .768 & & & \\
\hline \multirow{4}{*}{$\begin{array}{c}\text { Weight of a } \\
\text { door }\end{array}$} & A & 3.27 & .837 & \multirow{4}{*}{0.943} & \multirow{4}{*}{0.420} & \multirow{4}{*}{-} \\
\hline & B & 3.05 & .696 & & & \\
\hline & $\mathrm{C}$ & 3.14 & .645 & & & \\
\hline & $\mathrm{D}$ & 3.08 & .652 & & & \\
\hline \multirow{4}{*}{$\begin{array}{c}\text { Door } \\
\text { satisfaction }\end{array}$} & A & 3.51 & .898 & \multirow{4}{*}{1.963} & \multirow{4}{*}{0.121} & \multirow{4}{*}{-} \\
\hline & B & 3.25 & .650 & & & \\
\hline & $\mathrm{C}$ & 3.24 & .630 & & & \\
\hline & $\mathrm{D}$ & 3.17 & .678 & & & \\
\hline \multirow{4}{*}{ Extent } & A & 3.63 & .859 & & & \\
\hline & B & 3.19 & .752 & 3851 & $010 *$ & \\
\hline & $\mathrm{C}$ & 3.45 & .959 & 3.851 & .010 & - \\
\hline & D & 3.12 & .878 & & & \\
\hline & A & 3.51 & .840 & & & \\
\hline Height of a & $\mathrm{B}$ & 3.25 & .707 & 2353 & 0073 & \\
\hline $\operatorname{sink}$ & $\mathrm{C}$ & 3.47 & .844 & 2.353 & $0.0 / 3$ & - \\
\hline & D & 3.17 & .678 & & & \\
\hline & A & 3.49 & .840 & & & \\
\hline Faucet & $\mathrm{B}$ & 3.25 & .650 & 2405 & 0068 & 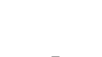 \\
\hline raucet & $\mathrm{C}$ & 3.49 & .794 & 2.400 & 0.068 & - \\
\hline & $\mathrm{D}$ & 3.17 & .760 & & & \\
\hline & A & 3.46 & .869 & & & \\
\hline Height of a & B & 3.25 & .650 & 1396 & 0245 & - \\
\hline toilet & $\mathrm{C}$ & 3.45 & .765 & 1.390 & & - \\
\hline & D & 3.23 & .807 & & & \\
\hline
\end{tabular}

Table 7. Continued.

\begin{tabular}{|c|c|c|c|c|c|c|}
\hline Factors & & Mean & S.D & $\mathrm{F}$ & Sig. & Scheffe \\
\hline \multirow{4}{*}{$\begin{array}{c}\text { Position of } \\
\text { the toilet } \\
\text { paper holder }\end{array}$} & A & 3.49 & .810 & \multirow{4}{*}{0.723} & \multirow{4}{*}{0.539} & \multirow{4}{*}{-} \\
\hline & B & 3.34 & .638 & & & \\
\hline & $\mathrm{C}$ & 3.27 & .811 & & & \\
\hline & $\mathrm{D}$ & 3.37 & .687 & & & \\
\hline \multirow{4}{*}{$\begin{array}{l}\text { Position of a } \\
\text { flush button }\end{array}$} & A & 3.51 & .711 & \multirow{4}{*}{1.387} & \multirow{4}{*}{0.248} & \multirow{4}{*}{-} \\
\hline & B & 3.27 & .693 & & & \\
\hline & $\mathrm{C}$ & 3.29 & .736 & & & \\
\hline & $\mathrm{D}$ & 3.42 & .723 & & & \\
\hline \multirow{4}{*}{$\begin{array}{l}\text { Method of a } \\
\text { flush button }\end{array}$} & A & 3.41 & .894 & \multirow{4}{*}{0.483} & \multirow{4}{*}{0.694} & \multirow{4}{*}{-} \\
\hline & B & 3.30 & .686 & & & \\
\hline & $\mathrm{C}$ & 3.45 & .679 & & & \\
\hline & $\mathrm{D}$ & 3.40 & .693 & & & \\
\hline \multirow{4}{*}{$\begin{array}{l}\text { Slipperiness } \\
\text { of the floor }\end{array}$} & A & 3.22 & .852 & \multirow{4}{*}{4.764} & \multirow{4}{*}{$0.003^{* *}$} & \multirow{4}{*}{-} \\
\hline & B & 2.95 & .575 & & & \\
\hline & $\mathrm{C}$ & 3.33 & .718 & & & \\
\hline & $\mathrm{D}$ & 2.90 & .664 & & & \\
\hline \multirow{4}{*}{$\begin{array}{c}\text { Floor } \\
\text { materials }\end{array}$} & A & 3.49 & .898 & \multirow{4}{*}{4.306} & \multirow{4}{*}{$0.006^{* *}$} & \multirow{4}{*}{-} \\
\hline & $\mathrm{B}$ & 3.10 & .441 & & & \\
\hline & $\mathrm{C}$ & 3.27 & .670 & & & \\
\hline & $\mathrm{D}$ & 3.08 & .589 & & & \\
\hline \multirow{4}{*}{$\begin{array}{c}\text { The position } \\
\text { of a flush } \\
\text { handle }\end{array}$} & A & 3.46 & .809 & \multirow{4}{*}{1.204} & \multirow{4}{*}{0.309} & \multirow{4}{*}{-} \\
\hline & B & 3.27 & .593 & & & \\
\hline & C & 3.27 & .670 & & & \\
\hline & D & 3.21 & .667 & & & \\
\hline \multirow{4}{*}{$\begin{array}{c}\text { A flush } \\
\text { handle easy } \\
\text { to hold }\end{array}$} & A & 3.46 & .809 & \multirow{4}{*}{1.565} & \multirow{4}{*}{0.199} & \multirow{4}{*}{ - } \\
\hline & B & 3.24 & .604 & & & \\
\hline & $\mathrm{C}$ & 3.31 & .619 & & & \\
\hline & $\mathrm{D}$ & 3.17 & .706 & & & \\
\hline
\end{tabular}

The mean difference is significant at the 0.01 lever. S.D $=$ Standard Deviation. Sig $=$ Significant.

A:Nishigahara, B:Nishiarai, C:Shindensakura, D:Nakameguro

In case of Nakameguro showing different attributes of users, it was found that the lower user evaluation of playground equipment than other parks was statistically significant.

3. Demands for facilities

In case of entrances, compared to almost no demand for wheelchairs, the demand for baby strollers was high(Table 10). This result can be attributed to the fact that the main user group was women accompanied by children. It is thus necessary to 
Table 8. Evaluation of park facilities and differences in evaluation for each park(Playground equipment).

\begin{tabular}{|c|c|c|c|c|c|c|}
\hline Factors & & Mean & S.D & $\mathrm{F}$ & Sig. & Scheffe \\
\hline \multirow{4}{*}{ Satisfaction } & $\mathrm{A}$ & 3.24 & 1.044 & \multirow{4}{*}{7.760} & \multirow{4}{*}{$0.000^{* *}$} & \multirow{4}{*}{$\mathrm{C}>\mathrm{D}$} \\
\hline & $\mathrm{B}$ & 3.51 & 1.048 & & & \\
\hline & $\mathrm{C}$ & 3.84 & .874 & & & \\
\hline & $\mathrm{D}$ & 2.96 & .791 & & & \\
\hline \multirow{4}{*}{ A view } & A & 3.95 & .865 & \multirow{4}{*}{5.901} & \multirow{4}{*}{$.001^{* *}$} & \multirow{4}{*}{$\mathrm{C}>\mathrm{D}$} \\
\hline & B & 3.91 & .950 & & & \\
\hline & C & 4.16 & .898 & & & \\
\hline & $\mathrm{D}$ & 3.42 & .936 & & & \\
\hline \multirow{4}{*}{$\begin{array}{c}\text { Space between } \\
\text { playground } \\
\text { equipment }\end{array}$} & A & 3.66 & .938 & \multirow{4}{*}{5.414} & \multirow{4}{*}{$0.001^{* *}$} & \multirow{4}{*}{$\mathrm{B}>\mathrm{D}$} \\
\hline & $\mathrm{B}$ & 4.09 & .865 & & & \\
\hline & $\mathrm{C}$ & 3.88 & 1.073 & & & \\
\hline & $\mathrm{D}$ & 3.46 & .803 & & & \\
\hline \multirow{4}{*}{$\begin{array}{c}\text { Space for } \\
\text { caring for a } \\
\text { playing child }\end{array}$} & A & 3.66 & 1.015 & \multirow{4}{*}{2.506} & \multirow{4}{*}{0.060} & \multirow{4}{*}{-} \\
\hline & B & 3.72 & 1.109 & & & \\
\hline & C & 3.71 & 1.258 & & & \\
\hline & D & 3.21 & 1.160 & & & \\
\hline \multirow{4}{*}{ Floor safety } & A & 2.90 & .944 & \multirow{4}{*}{15.221} & \multirow{4}{*}{$0.000^{* *}$} & \multirow{4}{*}{$\begin{array}{l}B>A \\
C>A \\
C>D\end{array}$} \\
\hline & B & 3.65 & .863 & & & \\
\hline & C & 4.06 & .801 & & & \\
\hline & $\mathrm{D}$ & 3.31 & .829 & & & \\
\hline \multirow{4}{*}{ Material safety } & A & 3.51 & .810 & \multirow{4}{*}{6.433} & \multirow{4}{*}{$0.000^{* *}$} & \multirow{4}{*}{$\begin{array}{l}B>D \\
C>D\end{array}$} \\
\hline & B & 3.86 & .873 & & & \\
\hline & $\mathrm{C}$ & 3.96 & .789 & & & \\
\hline & $\mathrm{D}$ & 3.37 & .715 & & & \\
\hline \multirow{4}{*}{ Sorts } & A & 2.27 & .923 & \multirow{4}{*}{7.106} & \multirow{4}{*}{$0.000^{* *}$} & \multirow{4}{*}{$\begin{array}{l}C>A \\
C>D\end{array}$} \\
\hline & B & 2.48 & .860 & & & \\
\hline & $\mathrm{C}$ & 3.04 & 1.172 & & & \\
\hline & $\mathrm{D}$ & 2.31 & .755 & & & \\
\hline \multirow{4}{*}{$\begin{array}{c}\text { Maintenance } \\
\text { status }\end{array}$} & A & 3.76 & .799 & & & \\
\hline & B & 3.72 & .831 & 6981 & $0.000^{* *}$ & $\mathrm{~B}>\mathrm{D}$ \\
\hline & $\mathrm{C}$ & 3.88 & .807 & 0.981 & 0.000 & $\mathrm{C}>\mathrm{D}$ \\
\hline & $\mathrm{D}$ & 3.23 & .614 & & & \\
\hline
\end{tabular}

The mean difference is significant at the 0.01 level. ${ }^{* *}$ S.D $=$ Standard Deviation. Sig $=$ Significant.

A:Nishigahara, B:Nishiarai, C:Shindensakura, D:Nakameguro

reset the guideline standards for wheelchairs to include strollers. There were more opinions about the sunlight measures than 'no particular demand' in all parks unlike on the entrance(Table 11).
Table 9. Evaluation of park facilities and differences in evaluation for each park(Bulletin board).

\begin{tabular}{|c|c|c|c|c|c|c|}
\hline Factors & & Mean & S.D & $\mathrm{F}$ & Sig. & Scheffe \\
\hline \multirow{4}{*}{ Usability } & A & 3.15 & .963 & \multirow{4}{*}{4.187} & \multirow{4}{*}{$0.007^{* *}$} & \multirow{4}{*}{$\mathrm{C}>\mathrm{B}$} \\
\hline & B & 3.03 & .800 & & & \\
\hline & $\mathrm{C}$ & 3.55 & .792 & & & \\
\hline & $\mathrm{D}$ & 3.17 & .785 & & & \\
\hline \multirow{4}{*}{ Height } & A & 3.44 & .709 & \multirow{4}{*}{1.786} & \multirow{4}{*}{0.151} & \multirow{4}{*}{$\mathrm{C}>\mathrm{D}$} \\
\hline & B & 3.42 & .727 & & & \\
\hline & $\mathrm{C}$ & 3.55 & .709 & & & \\
\hline & $\mathrm{D}$ & 3.23 & .675 & & & \\
\hline \multirow{4}{*}{ Size } & A & 3.34 & .855 & \multirow{4}{*}{1.321} & \multirow{4}{*}{0.268} & \multirow{4}{*}{$\mathrm{B}>\mathrm{D}$} \\
\hline & $\mathrm{B}$ & 3.33 & .780 & & & \\
\hline & $\mathrm{C}$ & 3.57 & .736 & & & \\
\hline & $\mathrm{D}$ & 3.31 & .701 & & & \\
\hline \multirow{4}{*}{ Letter Size } & A & 3.10 & .995 & \multirow{4}{*}{1.899} & \multirow{4}{*}{0.131} & \multirow{4}{*}{-} \\
\hline & $\mathrm{B}$ & 3.24 & .788 & & & \\
\hline & $\mathrm{C}$ & 3.49 & .767 & & & \\
\hline & $\mathrm{D}$ & 3.29 & .667 & & & \\
\hline \multirow{4}{*}{$\begin{array}{l}\text { Knowable } \\
\text { explanation }\end{array}$} & A & 3.51 & .840 & \multirow{4}{*}{6.313} & \multirow{4}{*}{$0.000^{* *}$} & \multirow{4}{*}{$\mathrm{C}>\mathrm{B}$} \\
\hline & $\mathrm{B}$ & 3.00 & .734 & & & \\
\hline & $\mathrm{C}$ & 3.51 & .767 & & & \\
\hline & $\mathrm{D}$ & 3.13 & .841 & & & \\
\hline \multirow{4}{*}{ Information } & A & 3.32 & .879 & \multirow{4}{*}{2.827} & \multirow{4}{*}{0.040} & \multirow{4}{*}{-} \\
\hline & $\mathrm{B}$ & 3.01 & .855 & & & \\
\hline & $\mathrm{C}$ & 3.43 & .791 & & & \\
\hline & $\mathrm{D}$ & 3.12 & .900 & & & \\
\hline
\end{tabular}

The mean difference is significant at the 0.01 level. ${ }^{*}$

S.D $=$ Standard Deviation. Sig $=$ Significant.

A:Nishigahara, B:Nishiarai, C:Shindensakura, D:Nakameguro

Contrary to the entrances that had the response of "no inconvenience', there were many opinions about sunlight measures in regard to paths. It also included various responses such as installation of garbage bins, increase of rest facilities, planting, and drainage. There were probably more demands because people spend more time on the path walking, exercising, and moving to other facilities. This is in contrast to entrances which are only used for entry to, and exit from the parks. Considering the characteristics of the elderly who are vulnerable to sunlight, it might be necessary to add standards in the guidelines for installing plants or facilities that can provide shade on the paths. 
Table 10. Demands about the entrance(\%).

\begin{tabular}{|c|c|c|c|c|}
\hline & A & B & $\mathrm{C}$ & $\mathrm{D}$ \\
\hline $\begin{array}{l}\text { Sunlight control } \\
\text { measure }\end{array}$ & 34.1 & 29.1 & 26.5 & 11.5 \\
\hline Garbage bins & 19.5 & 24.1 & 16.3 & 21.2 \\
\hline $\begin{array}{c}\text { Width for easy } \\
\text { passage of a wheelchair }\end{array}$ & 7.3 & 6.3 & 2.0 & 11.5 \\
\hline $\begin{array}{c}\text { Gradient for easy } \\
\text { passage of a wheelchair }\end{array}$ & 4.9 & 1.3 & 2.0 & 9.6 \\
\hline $\begin{array}{l}\text { Width for easy passage of a } \\
\text { stroller }\end{array}$ & 14.6 & 22.8 & 20.4 & 26.9 \\
\hline $\begin{array}{l}\text { Gradient for easy } \\
\text { passage of a stroller }\end{array}$ & 12.2 & 12.7 & 20.4 & 15.4 \\
\hline Lighting installation & 2.4 & 6.3 & 2.0 & 5.8 \\
\hline $\begin{array}{l}\text { Measures against } \\
\text { rainwater puddles }\end{array}$ & 17.1 & 7.6 & 2.0 & 9.6 \\
\hline $\begin{array}{c}\text { Mark noticeable from } \\
\text { outside }\end{array}$ & 4.9 & 7.6 & 6.1 & 9.6 \\
\hline $\begin{array}{c}\text { Measures for } \\
\text { prohibition of vehicular } \\
\text { access }\end{array}$ & 0.0 & 1.3 & 8.2 & 5.8 \\
\hline $\begin{array}{l}\text { Ensuring an outlook } \\
\text { that is visible when } \\
\text { entering and exiting }\end{array}$ & 7.3 & 2.5 & 6.1 & 1.9 \\
\hline $\begin{array}{l}\text { Signage which is easy } \\
\text { to see and understand }\end{array}$ & 2.4 & 3.8 & 2.0 & 11.5 \\
\hline No particular demand & 26.8 & 19.0 & 32.7 & 26.9 \\
\hline Others & 4.9 & 2.5 & 2.0 & 0.0 \\
\hline
\end{tabular}

A:Nishigahara, B:Nishiarai, C:Shindensakura, D:Nakameguro

In case of restrooms(Table 12), there was a high demand for facility cleanliness. This is similar to the results on maintenance status, which was the main factor influencing satisfaction with the use of public restrooms in Korean national parks(Lee et al., 2011). Since unclean facilities can be a barrier that can prevent users to use the restrooms, there is a need to add standards of maintaining cleanliness to the guidelines.

Secondly, the demand for security in crime-prevention measures can be said to coincide with the argument advocated by Rho et al.(1995). That guaranteeing security need, which is the sub-hierarchical structure of a user's needs, is an effective scheme that could raise the satisfaction with the use of a park. We need to have guideline standards that can increase not only user convenience, but also security. Measures of cleanliness and crime prevention are matters on maintenance/management that should be added to the guidelines. On playground equipment,
Table 11. Demands about the path(\%)

\begin{tabular}{|c|c|c|c|c|}
\hline & A & B & $\mathrm{C}$ & $\mathrm{D}$ \\
\hline $\begin{array}{l}\text { Sunlight control } \\
\text { measure }\end{array}$ & 58.5 & 63.3 & 49.0 & 48.1 \\
\hline Planting along the long path & 7.3 & 3.8 & 16.3 & 9.6 \\
\hline Flower bed & 4.9 & 11.4 & 8.2 & 5.8 \\
\hline $\begin{array}{l}\text { Drain facility not } \\
\text { inconveniencing passing }\end{array}$ & 2.4 & 1.3 & 6.1 & 3.8 \\
\hline Garbage bins & 19.5 & 20.3 & 26.5 & 25.0 \\
\hline $\begin{array}{l}\text { Bulletin board with } \\
\text { facilities information or events } \\
\text { announcements }\end{array}$ & 0.0 & 6.3 & 2.0 & 1.9 \\
\hline $\begin{array}{l}\text { Measures against } \\
\text { rainwater puddles }\end{array}$ & 12.2 & 13.9 & 12.2 & 5.8 \\
\hline $\begin{array}{l}\text { Extension in-between } \\
\text { rest facility }\end{array}$ & 12.2 & 24.1 & 42.9 & 26.9 \\
\hline Lighting & 12.2 & 6.3 & 4.1 & 11.5 \\
\hline $\begin{array}{l}\text { Easy to understand } \\
\text { lines }\end{array}$ & 0.0 & 2.5 & 0.0 & 1.9 \\
\hline No particular demand & 24.4 & 16.5 & 14.3 & 17.3 \\
\hline Others & 2.4 & 5.1 & 4.1 & 7.7 \\
\hline
\end{tabular}

A:Nishigahara, B:Nishiarai, C:Shindensakura, D:Nakameguro

Table 12. Demands about the restroom(\%).

\begin{tabular}{|c|c|c|c|c|}
\hline & $\mathrm{A}$ & $\mathrm{B}$ & $\mathrm{C}$ & $\mathrm{D}$ \\
\hline $\begin{array}{c}\text { Two separate restrooms for men } \\
\text { and women }\end{array}$ & 24.4 & 19.0 & 10.2 & 28.8 \\
\hline Soap availability & 12.2 & 13.9 & 32.7 & 21.2 \\
\hline Diaper change stand for adults & 2.4 & 0.0 & 0.0 & 3.8 \\
\hline Clean toilet & 29.3 & 44.3 & 57.1 & 34.6 \\
\hline Clean sink & 24.4 & 32.9 & 51.0 & 28.8 \\
\hline Clean diaper exchanger & 19.5 & 30.4 & 32.7 & 28.8 \\
\hline $\begin{array}{l}\text { Cleaning check list placed in an } \\
\text { easy to see location }\end{array}$ & 2.4 & 1.3 & 10.2 & 1.9 \\
\hline Easy to push call button & 7.3 & 1.3 & 8.2 & 5.8 \\
\hline Chair for children use & 22.0 & 22.8 & 28.6 & 19.2 \\
\hline Braille signage & 2.4 & 0.0 & 0.0 & 0.0 \\
\hline Security measures & 14.6 & 22.8 & 36.7 & 30.8 \\
\hline $\begin{array}{l}\text { Facilities information } \\
\text { board }\end{array}$ & 2.4 & 0.0 & 4.1 & 5.8 \\
\hline Installed in an easy to find place & 9.8 & 5.1 & 2.0 & 7.7 \\
\hline Installed in an easy to access place & 4.9 & 1.3 & 6.1 & 7.7 \\
\hline No particular demand & 46.3 & 38.0 & 22.4 & 19.2 \\
\hline Others & 4.9 & 3.8 & 0.0 & 5.8 \\
\hline
\end{tabular}

A:Nishigahara, B:Nishiarai, C:Shindensakura, D:Nakameguro 
Table 13. Demands about the playground equipment(\%).

\begin{tabular}{|c|c|c|c|c|}
\hline & A & $\mathrm{B}$ & $\mathrm{C}$ & $\mathrm{D}$ \\
\hline More variety & 48.8 & 53.2 & 30.6 & 34.6 \\
\hline $\begin{array}{l}\text { Exciting amusement } \\
\text { rides and tools though a little } \\
\text { challenging/dangerous }\end{array}$ & 9.8 & 7.6 & 8.2 & 11.5 \\
\hline $\begin{array}{l}\text { Fail proof safety } \\
\text { measures }\end{array}$ & 7.3 & 26.6 & 28.6 & 11.5 \\
\hline $\begin{array}{l}\text { Resting space while } \\
\text { caring for a child }\end{array}$ & 34.1 & 39.2 & 53.1 & 38.5 \\
\hline $\begin{array}{l}\text { Cleanliness } \\
\text { maintenance }\end{array}$ & 22.0 & 30.4 & 32.7 & 32.7 \\
\hline Flat floor & 9.8 & 8.9 & 4.1 & 11.5 \\
\hline $\begin{array}{l}\text { Space where one can } \\
\text { talk to guardians }\end{array}$ & 7.3 & 6.3 & 10.2 & 9.6 \\
\hline Lighting & 4.9 & 8.9 & 2.0 & 7.7 \\
\hline $\begin{array}{l}\text { Easy access to a diaper } \\
\text { changer }\end{array}$ & 7.3 & 16.5 & 6.1 & 7.7 \\
\hline $\begin{array}{l}\text { Easy access to a } \\
\text { restroom }\end{array}$ & 14.6 & 3.8 & 10.2 & 5.8 \\
\hline Easy access to a water stand & 7.3 & 5.1 & 6.1 & 5.8 \\
\hline $\begin{array}{l}\text { Measures that are } \\
\text { separated from other } \\
\text { park facilities users }\end{array}$ & 0.0 & 2.5 & 2.0 & 1.9 \\
\hline No particular demand & 24.4 & 12.7 & 20.4 & 23.1 \\
\hline Others & 9.8 & 6.3 & 2.0 & 3.8 \\
\hline
\end{tabular}

A:Nishigahara, B:Nishiarai, C:Shindensakura, D:Nakameguro

apart from 'more types of play equipments', there was a high demand for 'resting space while watching children'(Table 13). As most of the park users are mothers accompanied by children, it is necessary to have standards in which guardians can use playground equipment easily. Play guidelines describing only standards for safe playground equipments are insufficient. Playground equipments themselves should be included in the contents of the guidelines. Secondly, it is necessary for spatial planning to make it easier to take rest while watching children, use the restrooms, and change diapers. Thirdly, challenge taking, or little danger that is possible to evade is one of the intended purposes of amusement rides and tools, though guardians showed a tendency to think much about safety. Guardians accompanied by infants aged two to three showed the use behavior of concern for their children's safety near the amusement rides and tools. It is necessary to reflect the spatial scope in designing amusement rides and tools, through
Table 14. Demands about the bulletin board(\%).

\begin{tabular}{|c|c|c|c|c|}
\hline & A & $\mathrm{B}$ & $\mathrm{C}$ & $\mathrm{D}$ \\
\hline Lighting & 19.5 & 6.3 & 6.1 & 7.7 \\
\hline Sunlight control measure & 14.6 & 7.6 & 8.2 & 9.6 \\
\hline Cleanliness maintenance & 22.0 & 19.0 & 16.3 & 21.2 \\
\hline Surface without glare & 7.3 & 7.6 & 8.2 & 9.6 \\
\hline Braille signage & 2.4 & 2.5 & 4.1 & 0.0 \\
\hline $\begin{array}{l}\text { Measures against } \\
\text { fading bulletin board color }\end{array}$ & 0.0 & 16.5 & 10.2 & 17.3 \\
\hline No particular demand & 43.9 & 53.2 & 63.3 & 50.0 \\
\hline Others & 2.4 & 2.5 & 0.0 & 1.9 \\
\hline
\end{tabular}

A:Nishigahara, B:Nishiarai, C:Shindensakura, D:Nakameguro

Table 15. Demands about the information(\%).

\begin{tabular}{|c|c|c|c|c|}
\hline & A & $\mathrm{B}$ & $\mathrm{C}$ & $\mathrm{D}$ \\
\hline General view & 19.5 & 20.3 & 16.3 & 36.5 \\
\hline $\begin{array}{c}\text { Guidance on slope } \\
\text { gradient }\end{array}$ & 9.8 & 3.8 & 6.1 & 7.7 \\
\hline $\begin{array}{l}\text { Restroom information in } \\
\text { regard to the disabled } \\
\text { and seniors }\end{array}$ & 9.8 & 3.8 & 4.1 & 19.2 \\
\hline Diaper changing spots & 12.2 & 22.8 & 16.3 & 23.1 \\
\hline Barrier free map & 9.8 & 3.8 & 8.2 & 13.5 \\
\hline Guidance on facilities & 4.9 & 7.6 & 6.1 & 13.5 \\
\hline Events & 14.6 & 15.2 & 10.2 & 15.4 \\
\hline Volunteer activities & 9.8 & 2.5 & 4.1 & 11.5 \\
\hline $\begin{array}{l}\text { Measures against } \\
\text { accidents }\end{array}$ & 7.3 & 15.2 & 4.1 & 1.9 \\
\hline No particular demand & 53.7 & 39.2 & 49.0 & 21.2 \\
\hline Others & 2.4 & 6.3 & 2.0 & 3.8 \\
\hline
\end{tabular}

A:Nishigahara, B:Nishiarai, C:Shindensakura, :Nakameguro

research on children's evasive ability or the scope that enables adults to prevent an accident in advance by responding to children in danger.

There were no many demands for bulletin boards. It may be necessary to maintain them in a clean status, and to manage bulletin boards so that their colors do not fade(Table 14).

As for the requests on contents posted on the board, the above results highlights that there appeared a high response rate of 'no particular demand'in all parks but Nakameguro (Table 15). Such a high response rate can be attributed to the fact that the target park is located near users' residences. By the nature of the neighborhood park as the most basic park, most 
users answered 'no inconvenience', and that the park is of easy to use size and circulation system even without special information. Secondly, there was a lot of opinion in regard to request for information about the facilities in the premises of the park. Demand for information on location of diaper changing place, especially as the user comments were related to the park property. Thus, it is necessary to constitute a standard that can convey information about facilities effectively to users of each park.

Based on the above results, we can conclude as follows. Though the BF-related factors did not have a huge influence on standards of selecting parks, the BF improved facilities received relatively high user evaluation. Also, there were differences in user evaluation on BF facilities, due to the attributes of users, behavior of use, and internal characteristics of parks. The BF improvement of park facilities based on the set standards to enhance park user convenience has an effect on improvement of park user convenience. Such improvements are suitable for expected users' behavior including attributes of neighboring residents and characteristics of parks. Focusing on items showing different evaluations for each park such as the slope of the entrance or width of the path might be more effective to increase user convenience, rather than applying the same standards to every park. Considering the high ratio of female park users accompanied by children, there was a wide use of playground equipment in every park except for Nakameguro. There was a lot of opinion about placing facilities around playground equipment. These can be placed based on users' behavior and characteristics, and by including playground equipment into the contents of the guidelines. It would be necessary to add not only standards of design and construction, but also a manual of maintenance/management. In addition standards that can efficiently deliver information necessary for use of internal facilities of parks should also be prepared.

From the results, we make the following suggestions in order to improve user convenience of urban parks in Korea. It is necessary to have BF improvement of park facilities, to place facilities suitable for expected users' behavior, and characteristics of parks based on defined standards. It is also necessary to create efficient management regime to maintain clean facilities and understand the information desired by park users.

The results from analysis of the data received through the survey and suggestions can be summarized as follows.

Though the BF improvement of park facilities with set standards has an effect on improvement of user convenience, improvements suitable for the expected users' behavior, attributes of neighboring residents, and characteristics of parks can be more efficient to increase user convenience, rather than applying the same standards to every park. Secondly, it is necessary to place facilities depending on users' behavior and characteristics, and by including playground equipments into the contents of the guidelines.

\section{References}

Kang, B.K., K.J. Park, H.J. Chung, J.H. Kang, and J.H. Lee. 2007. A study on the planning of the urban park with analyse of barrier free urban parks in Japan. Architectural Institude of Korea Plan. 23(12):95-102.

Kawauchi, Y.H. 2001 Universal design for a question to barrier-free. 4 th ed. $7-8$.

Kim, S.H. 2006. Busan urban park development and citizen participation. Busan development forum. 98:13-18.

Lee, H., S.R. Lee, K.H. Park, E.H. park, J.Y. Cha, K.W. Sim, and D.W. Gang. 2011. A Study on visitor's perception of public toilet in the national park : focused on the Jirisan national park. Journal of National Park Research 2(1):1-5.

Lee, J.W and S.J. Yoem. 2012. A scheme proposal for barrier free investigation in maintenance of urban park in Korea for Japanese cases. J. Korean Soc. People Plants Environ. 15(3):191-199.

Lee, K.C. and K.W. Kim. 2001. A study on development priority of undeveloped urban neighborhood park in case of Daegu metropolitan city. Journal of the Korean Institute of Landscape Architecture 29(1):80-91.

Nishimura, M.J and T.Z. Asazawa. 2005. Issues related to barrier-free city park based on the new barrier-free law. Parks and Open Space Research Institute report 39-49.

Rho, J. H., J. Huh and D.S. Ahn. 1995. Perception of public safety of night users in neighborhood parks. Journal of National Park Research 23(2): 2213-2222.

Shigeno, T.M. 2008. An attempt to support the life and living of the elderly living alone : Reports from Kobe silver housing project. Review of Kobe Shinwa Women's University 41:99-107.

Suzuki, K.I. 2006. From barrier-free to universal design; Review of the traffic barrier-free law. Issue brief 526:1-9.

Wakisaka, R.I. 2008. Action for the barrier-free and the universal design in the urban park. Monthly construction opinion 15(2):28-31.

Guideline for promoting barrier-free transport and facilities for 
elderly and disabled on the city parks. 2008. Ministry of land, infrastructure, transport, and tourism.

\section{$\mathrm{V}$. 적요}

본 연구는 도시공원의 이용 편의성을 촉진시키기 위한 공원시 설의 베리어 프리화 정비 효과 검증 및 방향성을 제시하기 위해 일 본 도시 공원 가이드라인의 기준에 의해 설계·시공된 공원의 실제 공원 이용자들 이용 평가 및 의식조사를 실시하였다. 조사를 통해 얻은 자료를 분석한 결과 및 제안 사항을 정리하면 다음과 같다. 첫
째 구체적인 기준을 갖고 실시한 공원 시설의 베리어 프리화 정비 는 이용 편의성 향상이라는 효과가 있지만 똑같은 기준을 모든 공 원에 적용하기 보다는 공원의 특성과 인근 주민의 속성 등, 예상되 는 이용자의 이용행태에 맞게 정비하는 것이 이용자의 편의성을 높 이는데 유효하다. 둘째 가이드라인의 대상외인 유구도 가이드라인 의 대상 시설에 포함시켜 이용자의 이용 행태 및 특성을 고려해 시 설물을 배치할 필요가 있다. 설계 시공에 관한 기준뿐만 아니라 유 지관리에 관한 매뉴얼을 가이드라인에 추가할 필요가 있다. 공원 내부 시설 이용에 필요한 정보 내용 및 이를 효과적으로 전달 할 수 있는 기준마련이 필요하다. 